\title{
Radiotherapy in benign orbital disease. II: Ophthalmic Graves' disease and orbital histiocytosis $\mathrm{X}$
}

\author{
A N HARNETT, D DOUGHTY, A HIRST, AND P N PLOWMAN \\ From the Department of Radiotherapy, St Bartholomew's Hospital, London EC1
}

SUMmary Ophthalmic Graves' disease and histiocytosis X involving the orbit are occasionally refractory to treatment, so that vision may be threatened. In these situations megavoltage external beam radiotherapy should be employed, and the indications for this treatment are discussed. A highly accurate technique is described, using precise planning with information obtained from high definition CT scans, a complete patient head shell for immobilisation, and modern megavoltage radiotherapy treatment machines. As a result the dose to the lens is minimised (to a maximum of $10 \%$ of the prescribed dose), and late morbidity will be small. Two cases are described to illustrate this procedure and the response to treatment.

It is accepted practice that radiotherapy should be confined to the treatment of malignant disorders apart from exceptional circumstances. There exist benign orbital disorders which prove refractory to other treatments and, when imminently threatening vision, are well treated by low-dose external beam radiotherapy to the orbits. In such cases the radiotherapist is under a strict obligation to minimise any possible late tissue morbidity.

Orbital Graves' disease is a poorly understood condition that responds to low-dose orbital radiotherapy. The orbit and extraocular muscles are infiltrated by inflammatory cells, notably lymphocytes, and it is the exquisite sensitivity of the lymphocyte to steroids and radiation that is the rationale for this immunosuppressive treatment. The initial studies employed radiotherapy directed at the pituitary orbital regions using conventional orthovoltage equipment. ${ }^{12}$ The poorly collimated orthovoltage beams, with their considerable penumbra together with crude treatment planning, led to effectively large treatment portals being used. With modern radiotherapy for ophthalmic Graves' disease it is the tight collimation of the beam and the extreme accuracy of beam position that minimise late morbidity. Megavoltage radiotherapy was first employed for ophthalmic Graves' disease in $1973,{ }^{3}$

Correspondence to Dr P N Plowman, Department of Radiotherapy, St Bartholomew's Hospital, West Smithfield, London EC1A 7BE. and it is this Stanford technique that has laid the foundations of the modern techniques, one development of which is described here.

Another aetiologically obscure condition is histiocytosis $\mathrm{X}$, which is no longer considered to be a malignant tumour. ${ }^{+5}$ The bones of the skull are a site of predilection, and occasional cases of complicated orbital disease occur. A considerable body of literature has now recorded the efficacy of low-dose radiotherapy in such cases. ${ }^{5-7}$ Orbital radiotherapy is indicated where steroids have proved unsuccessful in arresting progressive orbital histiocytosis $\mathrm{X}$ and probably before administering systemic mutagenic chemotherapy for a local problem.

We here describe the technique used at St Bartholomew's Hospital to treat patients with orbital Graves' disease and histiocytosis X refractory to other forms of therapy and illustrate the technique with clinical examples of both conditions.

\section{Material and methods}

\section{TECHNIQUE}

Orbital radiotherapy is applied with a similar technique for both ophthalmic Graves' disease and histiocytosis X.

A high-definition computer tomography (CT) scan of the orbits is obtained before planning the radiotherapy. Cursor measurements from the lids to the 
back of the lens and the back of the globe aid planning. Standard tables of ocular dimensions supplement these data. ${ }^{\times}$

A supine plastic immobilisation head shell is made for each patient. This two-part device provides rigid confinement of the patient's head throughout the therapy session. Bilateral opposed temporal beam portals are planned on the treatment simulator. The superior and inferior field borders are defined by the orbital bony margins, and the posterior volume is set at the back of the orbital cone. The anterior field border is ascertained retrospectively by the planning constraint that the $10 \%$ isodose must be the maximum dose received at the back of the lens. A transverse section of the patient's head is taken at the level of the canthi with the lids closed; the positions of the orbits are marked in accord with CT data. Dose distribution (isodose) from the radiation portals is superimposed on this section, see Figs. 2 and 4. The lateral $6 \mathrm{MV}$ photon beams are angled posteriorly by their divergent angle so that the anterior field borders are parallel to the back of both lenses. In addition, to aid dose homogeneity, the fields are 'wedged', accommodating the change in patient contour anteriorly.

It is recognised that in order to spare the lens anteriorly sited swellings (notably the oedematous upper lid so common in ophthalmic Graves' disease) will not be encompassed within the primary beam. In Graves' disease placement of the posterior field border is influenced further by the requirement to retain pituitary dose level below $50 \%$ of the prescribed dose. Routine thermoluminescent dosimeters are employed on the lids of both eyes to check the dose is minimal in these regions.

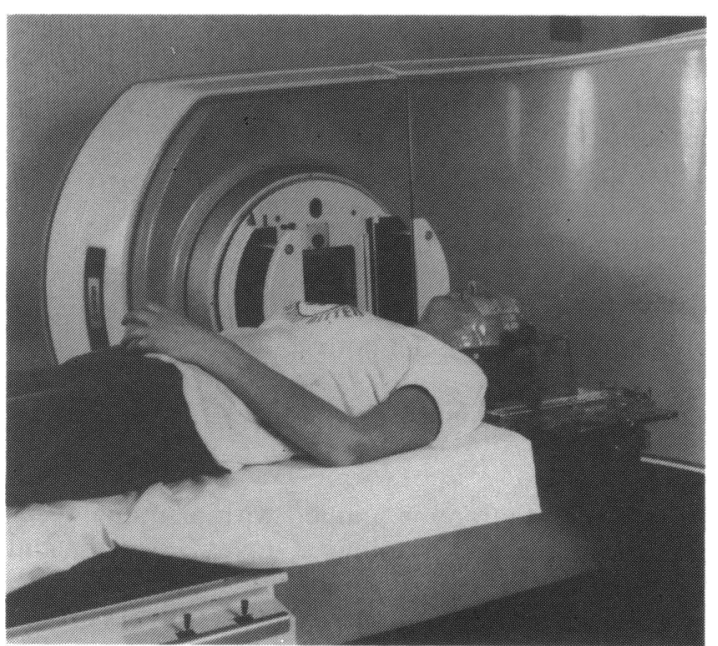

Fig. 1 Patient (case 1) set up for radiotherapy. Note immobilisation head shell and treatment planning marks.

\section{CASE REPORTS}

Case 1

This 32-year-old district nurse developed thyrotoxicosis three years earlier which was poorly controlled on carbimazole but responded well to partial thyroidectomy. For nine months she had developed diplopia with painful and 'bulging' eyes; this last complaint had become very much worse in the months leading up to her presentation. High-dose systemic steroids failed to control the condition. Surgical orbital decompressions brought temporary improvement, but the symptoms returned after three months. The patient was accepted for radiotherapy. At the time of therapy severe diplopia, proptosis, exposure keratitis, and impaired visual acuity (6/36 left eye and $6 / 24$ right) were present.

By the 6 MV $x$-ray technique described above, a prescribed dose of 2000 cGy was delivered in 12 fractions over 15 days (Figs. 1 and 2), with $100 \mathrm{cGy}$ being given through the lateral portals on the first day. The maximum dose to the lens was $200 \mathrm{cGy}$.

Visual acuities, which were monitored twice weekly, improved during radiotherapy. The patient's eyes became more comfortable and the diplopia improved. This was maintained and in fact continued at one year after treatment, with visual acuities of $6 / 9$ left and $6 / 6$ right.

\section{Case 2}

This 2-year-old girl presented with a history of an alternating convergent squint for one year and latterly a swelling over the left zygoma. As the swelling increased in size, the left eye became proptosed. There was a history of 'cradle cap' in infancy and discharging ears, the left worse than the right. From a biopsy from the scalp skin a diagnosis of histiocytosis $\mathrm{X}$ was made. Skull $x$-rays showed two well defined lytic lesions in the left parietal and frontal bones. Also on the plain radiograph there was widening and erosion of the floor of the pituitary fossa, with erosion of the floor of the anterior cranial fossa and of the left sphenoid wing and lateral wall of the orbit extending backwards. CT scanning showed the bony destruction as described, with increased soft tissue disease at the back of the left apex and extending across the midline behind both orbits. Visual acuity and the visual evoked responses were normal. The child developed diabetes insipidus, necessitating desmopressin acetate therapy. There was no response to systemic prednisolone.

There was concern that both optic nerves were at risk from the soft tissue retro-orbital disease and the larger bulk of destructive disease in the left orbit.

By the $6 \mathrm{MV} x$-ray technique described a prescribed dose of $1200 \mathrm{cGy}$ in seven fractions over eight days was delivered to the retro-ocular, orbital 


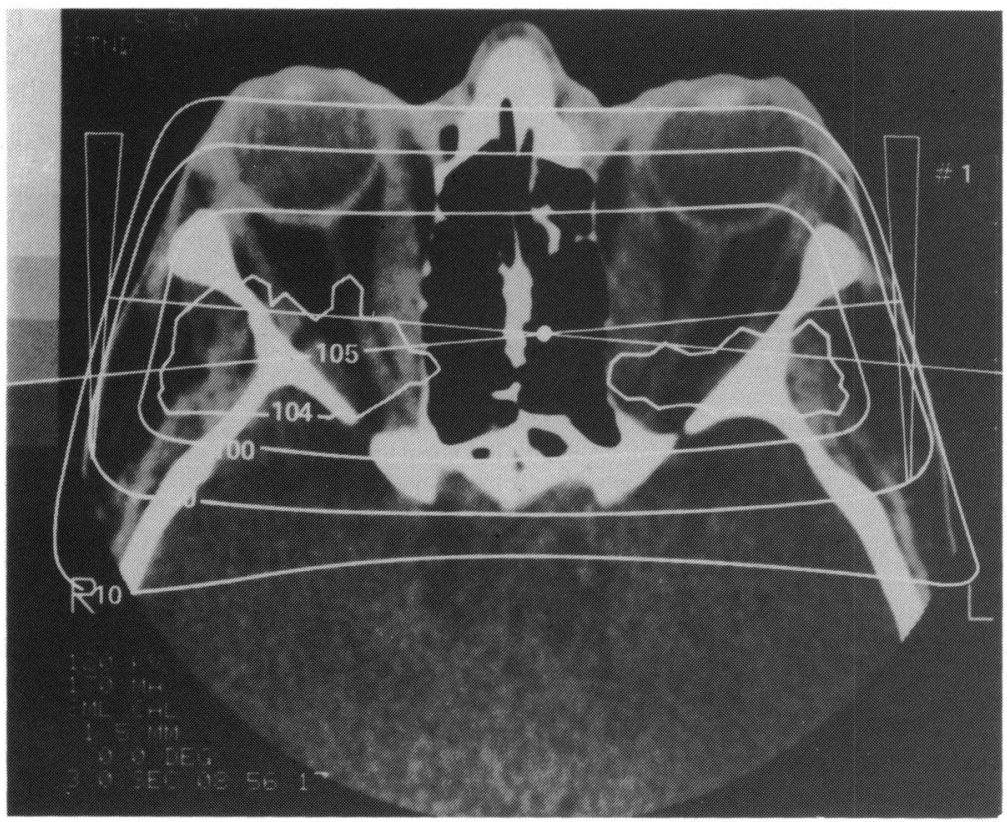

Fig. 2 CTscan of the orbits (case 1). Radiation isodoses are superimposed showing $10 \%$ isodose at back of lens and $100 \%$ encompassing the back of the orbital cone. Note also the greatly enlarged medial rectus muscles.

regions, the treatment volume extending further posteriorly than in case 1 to encompass the retroorbital soft tissue extension, hypothalamus, and pituitary (Figs. 3 and 4). However, a lower dose was employed.

The diabetes insipidus did not respond. The swelling in the left zygoma region and the proptosis regressed over a few months and remained so at one year. Magnetic resonance imaging at nine months after therapy confirmed less soft tissue disease. The child remains well.

\section{Discussion}

Traditional reluctance to employ radiotherapy for benign orbital disease has been based on anxiety concerning late morbidity, notably cataractogenesis. However, with modern radiotherapeutic techniques, well collimated $x$-ray beams can treat the orbit while keeping the lens below its cataract threshold. The technique described here relies on modern treatment equipment and on known depths of critical ocular points by high definition CT scanning with cursor measurements, supplemented by standard tables of ocular measurements. In addition, and critically, a head fixed by a full head shell allows reproducibility of positioning. These factors allow the dosimetric advance over previous treatment methods which historically used orthovoltage $x$-rays or telecobalt beams, the lateral skin canthus as the set-up mark for the anterior field edge or a bite block only to support the head.
In 1951 Jones" described a series of 29 patients with Graves' ophthalmopathy treated by radiotherapy at St Bartholomew's Hospital. He showed that it is the patients with fast, progressive, and serious disease that respond best to radiotherapy, an observation that has been confirmed by others. ${ }^{3}$ Glaser $^{10}$ considered that the indications for orbital radiotherapy in Graves' ophthalmopathy were only two: these are steroid refractory patients with 'documented optic neuropathy with visual acuity less than $20 / 40$ or an acute painful inflammatory phase of Graves' disease'. Our own indications also include pro-

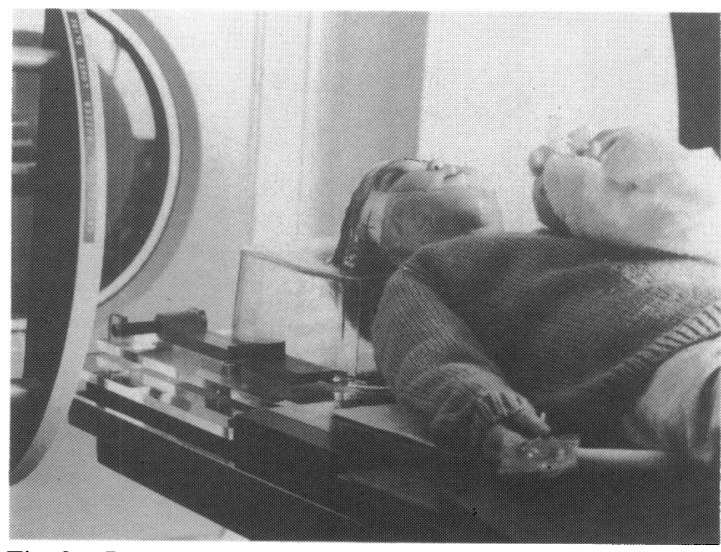

Fig. 3 Patient (case 2) set up for radiotherapy for orbital histiocytosis $X$. The immobilisation head shell is again important for precision treatment. 


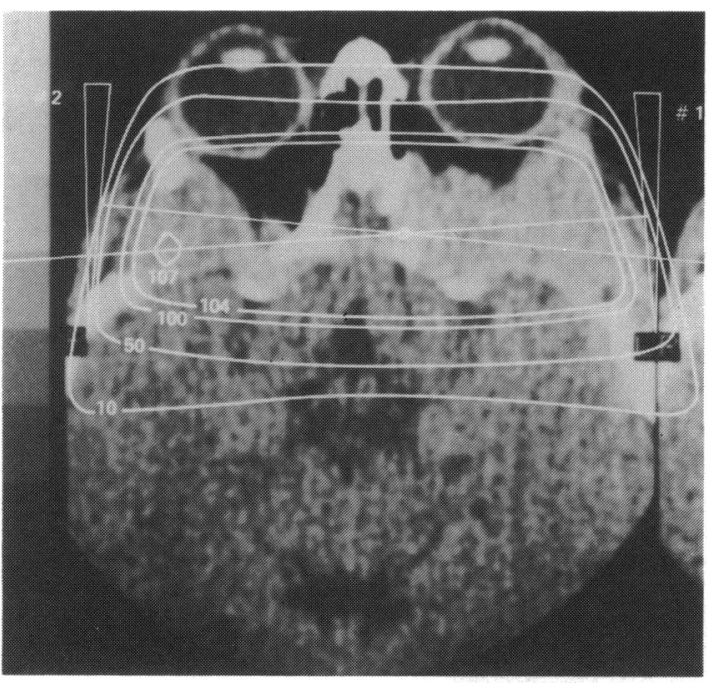

Fig. 4 CTscan of orbits (case 2). Note the extensive involvement of the posterior orbital tissues by histiocytosis $X$ is easily seen. Planning has been performed so that the $100 \%$ isodose includes the posterior aspect.

gressive diplopia or progressive exposure keratitis following failure of steroid therapy, but we agree with Glaser's general conclusion that the technique is not indicated for uncomplicated disease.

Similarly, the indications for radiotherapy in orbital histiocytosis $\mathrm{X}$ must be strict, but, as in the child reported on here, imminent visual compromise remains an important indication.

In conclusion, there remain complicated cases of benign orbital disease for which radiotherapy is indicated and in which, with techniques such as those described here, late morbidity will be small and the benefit to the patient immense.

It is with great pleasure that we thank Mr P Fells, Dr B Shine (Moorfields Hospital), Professor G M Besser (St Bartholomew's Hospital), and Dr J Pritchard (Hospital for Sick Children, London) for referring patients. Miss T Cocks kindly typed the manuscript.

\section{References}

1 Ginsburg S. Postoperative progressive exophthalmos, with low basal metabolic rate. Ann Intern Med 1939; 13: 424-50.

2 Mandeville FB. Roentgen therapy of orbital-pituitary portals for progressive exophthalmos following subtotal thyroidectomy. Radiology 1943; 41: 268-71.

3 Donaldson SS, Bagshaw MA, Kriss JP. Supervoltage orbital radiotherapy for Graves' ophthalmopathy. J Clin Endocrinol Metab 1973; 37: 276-85.

4 Osband ME, Lipton JM, Laving P, et al. Histiocytosis X. Demonstration of abnormal immunity. T cell histamine $\mathrm{H} 2$ receptor deficiency and successful treatment with thymic extract N Engl J Med 1981; 304: 146-53.

5 Nesbit ME. Current concepts and treatment of histiocytosis $X$ (Langerhans' cell histiocytosis). In: Voute PA, Barrett A, Bloom HJG, Lemerle J, Neidhardt MK, eds. Cancer in children. Berlin Springer, 1986: 176-84.

6 Childs DS, Kennedy RLJ. Reticuloendotheliosis of children: treatment with roentgen rays. Radiology 1951; 57: 653-9.

7 Ochsner SF. Eosinophilic granuloma of bone; experience with 20 cases. AJR 1966; 97: 719-26.

8 François J. Ocular biometry. Doc Ophthalmol Proc 1981; 29. $135-64$.

9 Jones AE. Orbital $x$-ray therapy of progressive exophthalmos. Br J Radiol 1951; 24: 637-46.

10 Glaser J. Editorial: Graves' ophthalmopathy. Arch Ophthalmol 1984; 102: 1148-9.

Accepted for publication 26 February 1987. 